\title{
Miniaturized Microwave Passive Filter Incorporating Multilayer Synthetic Quasi-TEM Transmission Line
}

\author{
Hsien-Shun Wu, Member, IEEE, Houng-Jay Yang, Ching-Juang Peng, and Ching-Kuang C. Tzuang, Fellow, IEEE
}

\begin{abstract}
This paper presents a novel approach to miniaturizing a transmission-line (TL)-based bandpass filter (BPF). The BPF is miniaturized by incorporating multilayer stacked synthetic quasi-TEM TLs made of complementary conducting strip (CCS) metal surfaces. The stacked CCS TLs are separated by a meshed ground plane, whose coupling effect was experimentally shown negligibly to affect desired circuit performance. A brief description of the symmetric third-order TL filter synthesis procedure is reported to map such an idealized BPF to the prototype made of the stacked synthetic CCS TLs. Excellent agreement is obtained by comparing the experimental and theoretical filter frequency responses showing a 2.46-dB insertion loss, a -16.8-dB return loss with under $5 \%$ offset of low-side out-band transmission zeros, and a $2 \%$ offset of center frequency. A quick estimate of the proposed miniaturized filter design based on synthetic quasi-TEM lines shows that the particular filter prototype approaches the process limit.
\end{abstract}

Index Terms-Bandpass filter (BPF), multilayer, multistandard, RF system-on-chip (SOC), synthetic transmission line (TL), system-in-package (SIP), wireless local area network (WLAN).

\section{INTRODUCTION}

$\mathbf{P}$ ORTABLE wireless devices have evolved into the convergence realm, integrating multifrequency and multistandard protocols, greatly enhancing the handheld devices' capabilities in managing voice and multimedia data. Smart phones, combining wireless local area network (WLAN) and cellular phones, are one example of this trend, which is driven by continuing technological development in the system-on-chip (SOC) and system-in-package (SIP). Although as many as possible wireless building blocks have been integrated into the SOC, antenna, filter, baluns, and other devices are mostly in discrete forms or embedded into the SIP. A complete solution for making a wireless device with a very small form factor is becoming a reality by combining advanced techniques of the SOC and SIP. Thus, this paper focuses on the miniaturization of the filter to the highest degree of integration density. What follows is the survey of filters, which include the state-of-the-art discrete fil-

Manuscript received January 20, 2005. This work was supported in part by the Ministry of Education of Taiwan under Grant NSC 93-2752-E-002-009-PAE and in part by Epic Communication Inc. under Grant 92C202.

H.-S. Wu is with the Electrical Communication Engineering Department, National Chiao Tung University, Hsinchu 300, Taiwan, R.O.C.

H.-J. Yang was with the Electrical Communication Engineering, National Chiao Tung University, Hsinchu 300, Taiwan, R.O.C.

C.-J. Peng is with Epic Communication Inc., Hsinchu 300, Taiwan, R.O.C.

C.-K. C. Tzuang is with the Graduate Institute of Communication Engineering, Department of Electrical Engineering, National Taiwan University, Taipei 106, Taiwan, R.O.C. (e-mail: cktzuang@cc.ee.ntu.edu.tw).

Digital Object Identifier 10.1109/TMTT.2005.854193 ters reported recently in the market, ${ }^{1234567891011}$ technical literature [1]-[23], and the filters in the advanced SIP [24]-[30].

Without loss of generality, Fig. 1 plots the thickness against volume for 2.4-GHz industrial-scientific-medical (ISM) bandpass filters (BPFs), allowing the assessment of the incorporation of filters in a specific user-defined environment to determine whether the filter with the small form factor is too thick or too thin to fit in the final integrated product. The square and triangular symbols represent filter with three and two reflection zeros in the passband, respectively. Closely examining the statistics shown in Fig. 1 supports the following summary. First, the volumes required to realize a $\mathrm{BPF}$ with three reflection zeros $\left(N_{\mathrm{RZ}}=3\right)$ are about one-half of those of a BPF with two reflection zeros. Second, these commercially available low-temperature co-fired ceramic (LTCC)-based filters contain approximately ten layers or more and, thus, are approximately $0.8-\mathrm{mm}$ thick in most designs. Third, transmission-line (TL)-based microwave filters [5]-[8] are normally large with volumes over $14 \mathrm{~mm}^{3}$; independent of the number of reflection zeros in the passband.

Fig. 1 also presents the sizes of the filters designed by the proposed integration method with various numbers of dielectric layers for two and three reflection zeros, respectively.

The BPF presented herein contains only TL elements to illustrate the core technique of incorporating synthetic quasi-TEM lines in the multilayer substrate environment. Synthetic quasi-TEM lines were recently reported for a miniaturized four-port rat-race device in printed circuit board (PCB) and a CMOS oscillator with a size comparable to those achieved

${ }^{1}$ LFB182G45SG9A246, Murata Manufact. Company, Koyoto, Japan. [Online]. Available: http://www.murata.com/

${ }^{2}$ DEA202450BT-1213C1, TDK Company Amer., Tokyo, Japan. [Online]. Available: http://www.component.tdk.com/

${ }^{3}$ LTF2012B-F2R4B, TOKO Amer. Inc., Mount Prospect, IL. [Online]. Available: http://www.tokoam.com/

${ }^{4}$ BF2012-F2R4DAA, Advanced Ceramic X Company, Hsinchu, Taiwan, R.O.C. [Online]. Available: http://www.acxc.com.tw/

52450BP15B100, Johanson Technol. Inc., Camarillo, CA. [Online]. Available: http://www.johansontechnology.com

${ }^{6}$ DEA252450BT-2027A1, TDK Company Amer., Tokyo, Japan. [Online]. Available: http://www.component.tdk.com/

${ }^{7}$ BF3216-B2R4BAA, Adv. Ceramic X Company, Hsinchu, Taiwan, R.O.C. [Online]. Available: http://www.acxc.com.tw/

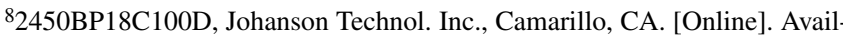
able: http://www.johansontechnology.com/

${ }^{9}$ B69812N2457C101, EPCOS, Munich, Germany. [Online]. Available: http://www.epcos.com/web/home/html/home_e.html

${ }^{10}$ LFS2450-01BB-C, NTK Technol. Inc., Chicago, IL. [Online]. Available: http://www.ntktech.com/

${ }^{11}$ LFB322G45SN1A504, Murata Manufact. Company, Kyoto, Japan. [Online]. Available: http://www.murata.com/ 


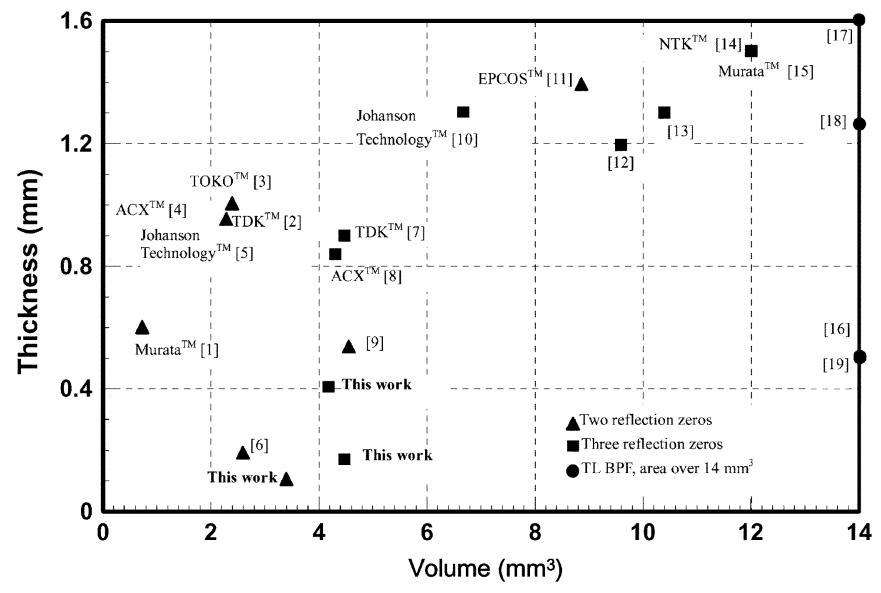

Fig. 1. Survey of 2.4-GHz ISM-band BPFs in size (volume) and thickness.

by the parallel inductor-capacitor approach [31]. This paper proposes a new approach to systematically reducing the size of filter based on multilayer substrate technology. The application of the synthetic quasi-TEM lines results in the filter designs of almost nearly the same volume as the number of substrates is increased. A designer can, therefore, optimize the area and thickness required for product integration. Section II will describe how to realize synthetic quasi-TEM lines in a multilayered substrate configuration. Followed by the measured validation that enough isolation can be achieved for various devices integrated in different layers, Section III reports the procedure for designing a TL filter to demonstrate the feasibility of incorporating the multilayer synthetic quasi-TEM line for making multilayered miniaturized filters. Section IV presents theoretical and measured results, indicating that the current approach meets the state-of-the-art size requirements for practical implementation in multistandard wireless product designs. Section V reports a quick estimate that the proposed integration method adopted for filter miniaturization approaches the process limit. Section VI concludes this paper.

\section{Multilayer Synthetic Quasi-TEM TL}

Recently, a new artificially engineered synthetic TL-the so-called complementary conducting strip transmission line (CCS TL) - was reported to be an effective means of miniaturizing microwave circuits [31]. The CCS TL has the following characteristics. It firstly provides wide design choices for making characteristic impedance of the TL without changing the process parameters and material constants. Second, the meandered CCS TL exhibits less bending and adjacent coupling effects, as indicated by the slower change in characteristic impedance against the width variation in the TL than the conventional meandered microstrip used in the same fashion [31, Fig. 5]. Therefore, a compact microwave circuit can be established using the meandered CCS TL, finally achieving miniaturization.

The CCS TL is made from a unit cell, which has dimensions that are much smaller than the operating wavelength. As shown in Fig. 2, a unit cell contains a mesh ground plane and a central patch with at least two series arms for cells in series [see Fig. 2(a)] and bent [see Fig. 2(b)] connection to the adjacent

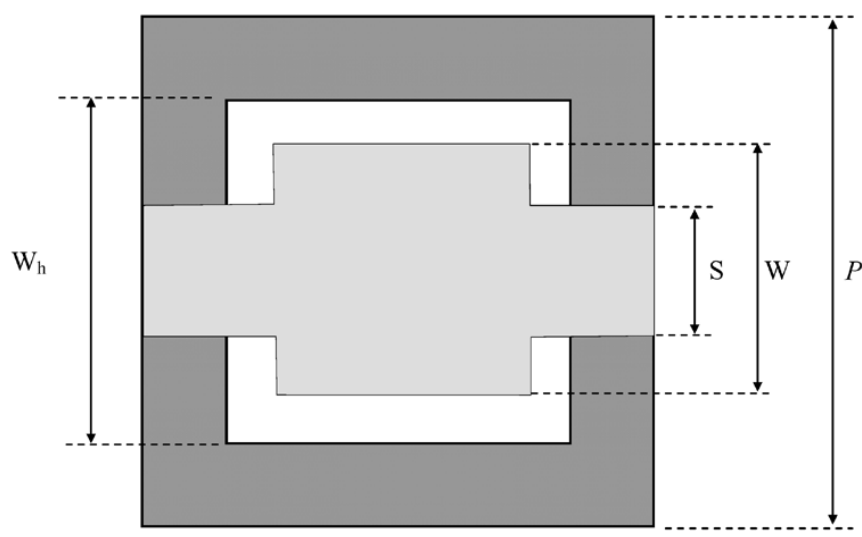

(a)

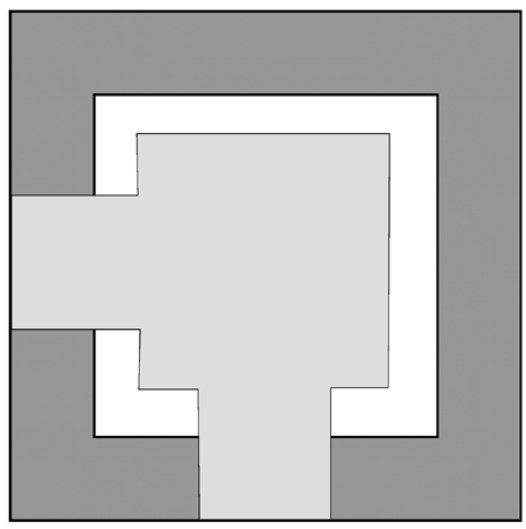

(b)

Fig. 2. Unit cells of CCS TL. (a) For series connection. (b) For bent connection.

cells. The etched portion of the meshed ground plane complements to the central patch of the signal layer, forming a CCS TL. Additionally, Fig. 3 shows a new multilayer meandered CCS TL configuration made of the meandered CCS TL realized by two metal layers [see Fig. 3(a)], whose guiding characteristics have been well documented [31], and the sandwiched CCS TL is realized by two meshed ground planes on the top and bottom surfaces [see Fig. 3(b)]. All the meshed ground planes are connected by plated through-vias. The procedure for designing a sandwiched CCS TL, however, is similar to the meandered CCS TL reported in [31], and will not be repeated here.

Notably, a four-layer substrate configuration was adopted throughout this paper. In such a configuration, Fig. 3(a) and (b) shares a common meshed ground plane M2. Based on this integration scheme, the CCS TLs in different layers can be independently controlled for various circuit designs. However, attention must be paid to the isolation of the stacked CCS TLs in different layers. The perfect solid ground plane provides the highest shielding capability of any mesh ground plane. An investigation on the shielding capability of two isolated circuits using meandered CCS TLs in different layers is followed.

Two filters with independent functions are designed using CCS TLs and integrated in the same four-layer substrate configuration. The first is the low-pass filter (LPF), which occupies M1 and M2 layers [see Fig. 3(a)]. The second is the BPF, which utilizes the M2-M4 layers [see Fig. 3(b)]. These symmetrical filters are designed following the similar procedure, which is 


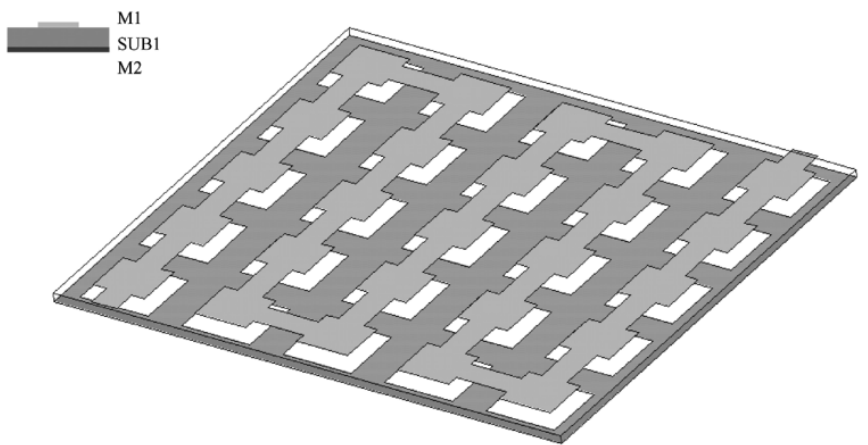

(a)

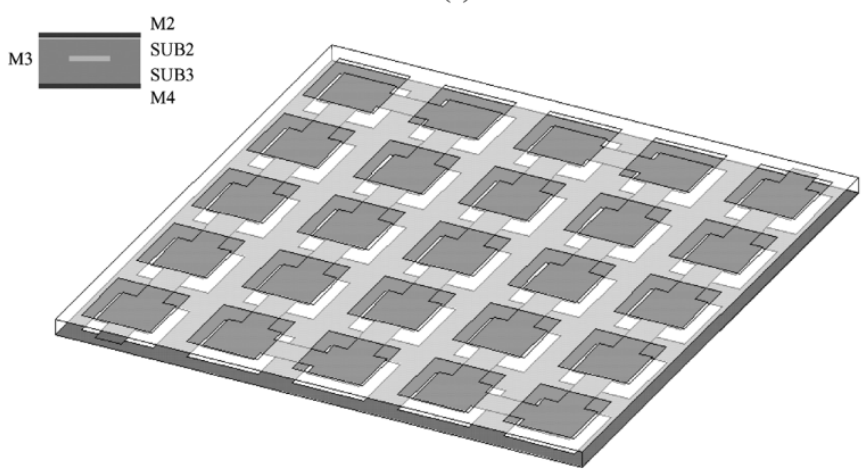

(b)

Fig. 3. Synthetic CCS TL. (a) Meandered CCS TL. (b) Sandwiched meandered CCS TL.

reported in Section III. In Fig. 3, every substrate has an equal thickness of $0.06 \mathrm{~mm}$ (SUB1-SUB3). The area of overlapping of the two filters is approximately $95 \%$ of the total area. Fig. 4(a) and (b) shows the intrinsic frequency responses, including experimental and theoretical results, for two standalone filters.

In the experiments, one filter is measured using the two-port vector network analyzer (VNA) and the other is terminated by two $50-\Omega$ chip resistors. The full-wave simulations using Zeland IE3D follow the same procedure. The cutoff frequency of the LPF is $2.75 \mathrm{GHz}$, and the out-of-band rejection is below $30 \mathrm{~dB}$ from 4.25 to $4.7 \mathrm{GHz}$. The insertion loss is approximately $0.92 \mathrm{~dB}$, a little higher than the simulated value of $0.45 \mathrm{~dB}$. The return loss is below $-10 \mathrm{~dB}$ from 2.38 to $2.51 \mathrm{GHz}$. On the other hand, in Fig. 4(b), the center frequency of the BPF is $2.51 \mathrm{GHz}$, and the return loss is below $-11.5 \mathrm{~dB}$ from 2.11 to $2.91 \mathrm{GHz}$. The measured insertion loss is approximately $1.48 \mathrm{~dB}$, which is $0.39 \mathrm{~dB}$ higher than the simulated value. Good agreement between the measurements and simulations for the two filters show that the structural parameters and material constants are very close to the design values. Additionally, the transmission between port 3 and either ports 1 or 2 is measured to evaluate the cross coupling between the LPF and BPF. Fig. 4(c) shows the measured transmission coefficient across two filters. Based on the measured results presented in Fig. 4(a) and (b), the BPF passes the energy above $2.11 \mathrm{GHz}$ with low reflection and the LPF rejects signals above $2.75 \mathrm{GHz}$. The electromagnetic (EM) energy can be distributed in the four-layer configuration from 2.11 to $2.75 \mathrm{GHz}$. Fig. 4(c) plots the measurements for the adjacent-port coupling $\left(\left|S_{32}\right|\right)$ and cross-port coupling $\left(\left|S_{31}\right|\right)$. The filter is symmetrical so only port 3 is applied when port 4 is terminated. Although Fig. 4(c) reveals the relatively high EM en-

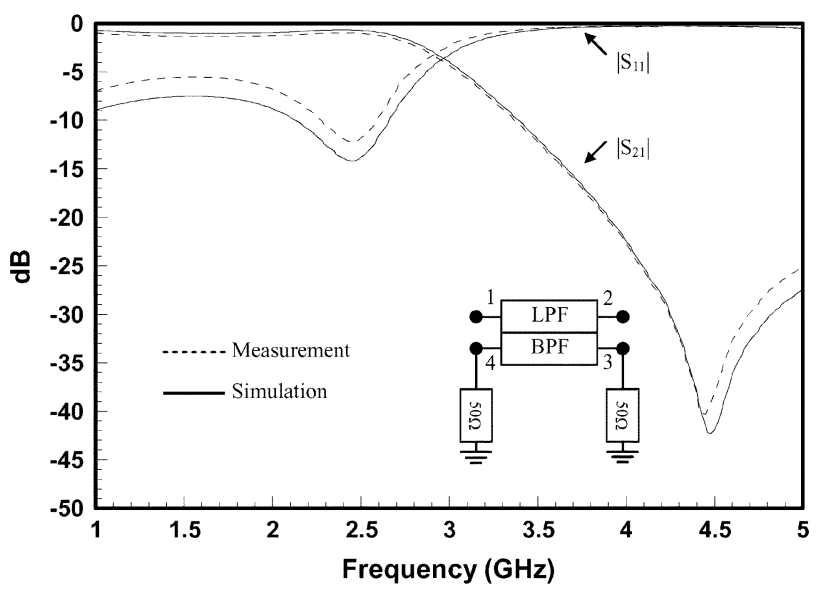

(a)

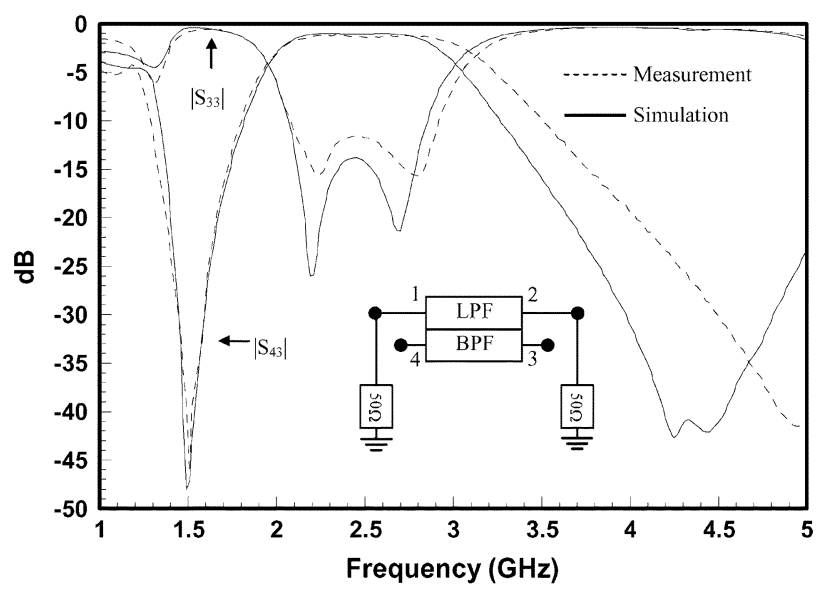

(b)

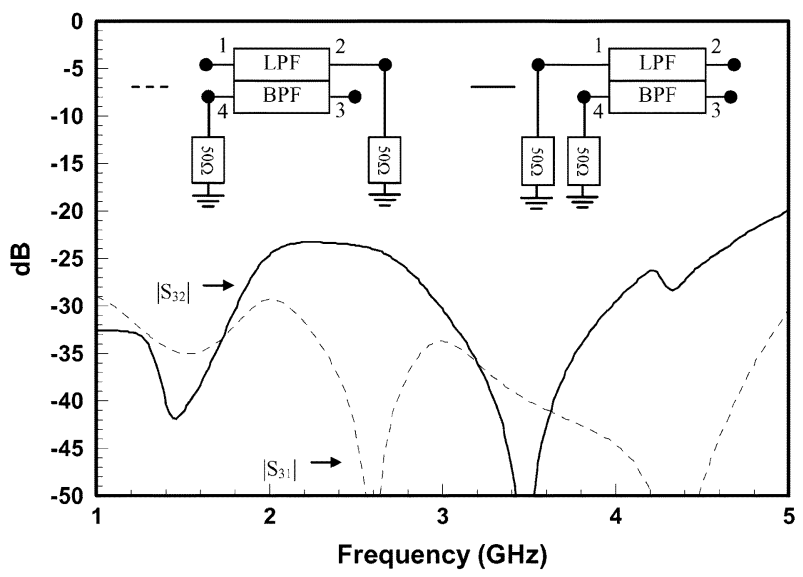

(c)

Fig. 4. Multifunction module incorporating four-layer meandered CCS TLs. (a) Frequency responses of 2.4-GHz LPF in M1 and M2 metal layers. (b) Frequency responses of $2.4-\mathrm{GHz}$ BPF in M2-M4 metal-layers. (c) Measured transmission coefficient between LPF and BPF in four-layer CCS TL's configuration.

ergy transmission between the two filters in different layers from $2.112 .75 \mathrm{GHz}$, to the adjacent coupling to is maintained below $-23 \mathrm{~dB}$ and the cross-coupling is below $-29 \mathrm{~dB}$. Therefore, Fig. 4(c) verifies that passive circuits realized by the stacked CCS TLs in different layers can be well isolated from each other. In the case study, an isolation of more than $23 \mathrm{~dB}$ is achieved. 


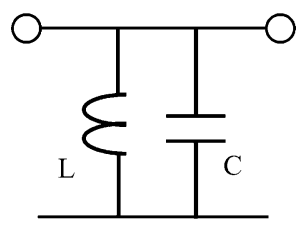

(a)

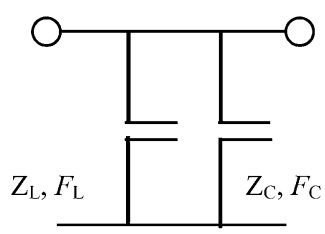

(b)

Fig. 5. Parallel resonators. (a) Lumped realization. (b) TL realization.

\section{EXPERIMENTAL FILTER Design Procedure}

Recently, Quendo et al. reported that a TL BPF, incorporating the so-called dual-behavior resonator (DBR), could achieve an $N$ th-order BPF with $2 N$ pre-selective transmission zeros using $2 N$ open and/or short stubs [32]-[34]. One DBR, which contains two open/short stubs of different lengths and characteristic impedances, independently controls two transmission zeros. The designs of such filters have been well documented [32]-[34]. This paper presents the similar filter architecture to demonstrate the filter miniaturization, incorporating meandered stacked CCS TLs. However, a larger $N$ th-order TL BPF with only $2(N-1)$ pre-selective transmission zeros using $2 N$ open stubs by adding an additional pair of shunt stubs and a TL connected in series is designed to achieve a symmetrical filter with a direct interface to external $50-\Omega$ loads.

The TL BPF design begins with the design of parallel resonators. Fig. 5 shows the equivalent circuit of the parallel resonator in both lumped [see Fig. 5(a)] and TL [see Fig. 5(b)] forms. $Z_{c}$ and $Z_{L}$ represent the characteristic impedances of the two TLs. $F_{c}$ and $F_{L}$ are the quarter-wavelength frequencies of the TLs.

Assuming that the TLs are lossless, the input susceptance of the resonator is given by the following equation:

$$
B_{\text {res }}=j\left[Y_{c} \tan \left(\theta_{c}\right)+Y_{L} \tan \left(\theta_{L}\right)\right] .
$$

Variables $\theta_{c}$ and $\theta_{L}$ are the electric lengths of the two TLs at the center frequency $\left(f_{0}\right)$ of the BPF. $Y_{c}$ and $Y_{L}$ represent the inverse of the characteristic impedances of the two TLs. If $F_{L}$ is defined below $f_{0}$, then $F_{c}$ must be above $f_{0}$. The susceptances of the open stub that is one quarter-wavelength frequency below (above) $f_{0}$ is negative (positive) near $f_{0}$, as shown in Fig. 6. The sum of the two curves indicates a parallel resonance at $f_{0}$. Therefore, $Z_{c}, Z_{L}, F_{c}$, and $F_{L}$ can be chosen to make the input susceptances of the parallel resonator zero at the $f_{0}$ of the BPF.

Next, the conventional LPF synthesis procedure is invoked for the TL BPF design for an $N$ th-order LPF prototype with a specified passband ripple, as shown in Fig. 7(a) [35]. Fig. 7(b) transforms the series inductor $\left(g_{2}\right)$ into a T-network of two $J$-inverters and one shunt capacitor after some algebraic manipulation and conversion [36]. The passband bandwidth and reference

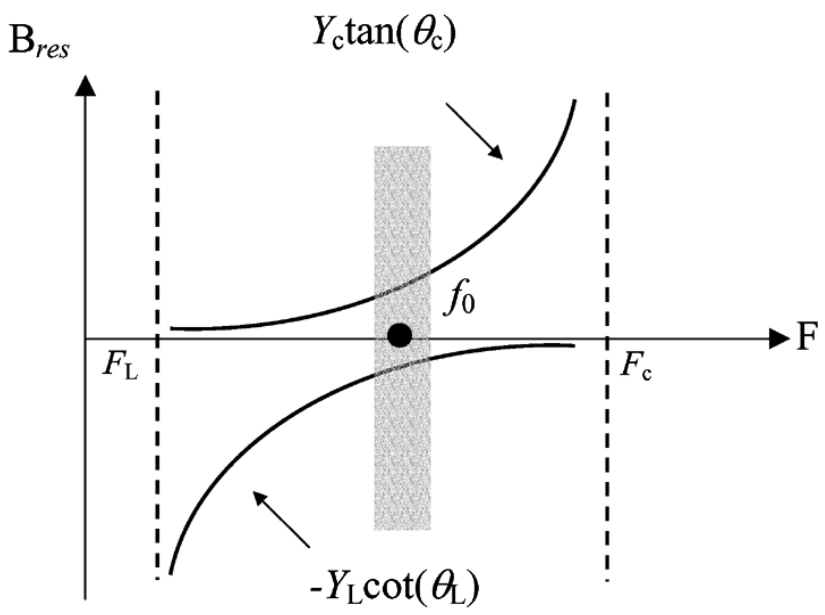

Fig. 6. Input susceptance of the parallel resonator using TL realization.

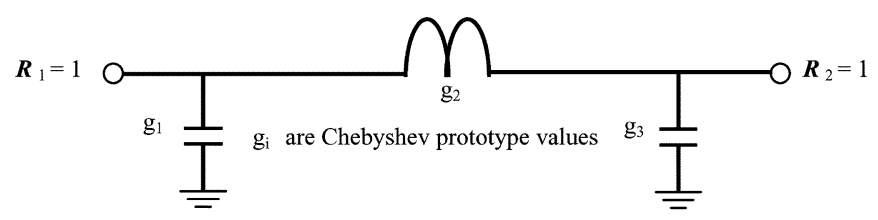

(a)

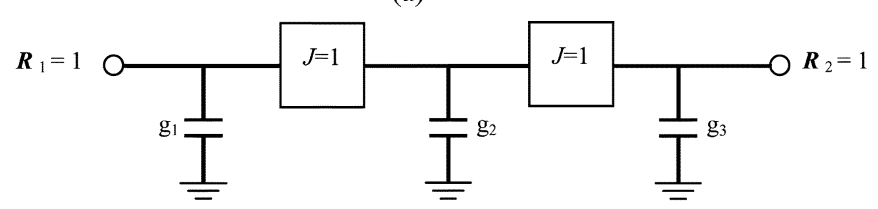

(b)

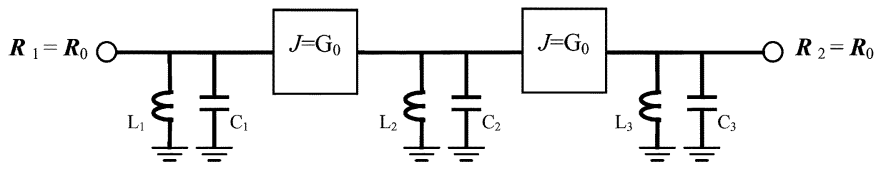

(c)

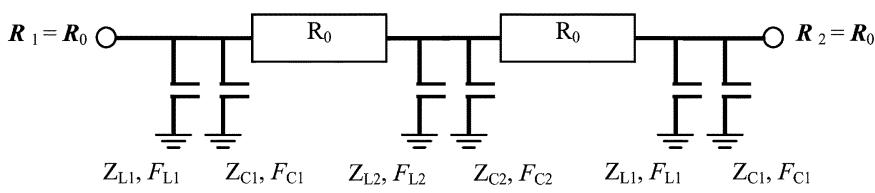

(d)

Fig. 7. Brief description of the TL BPF design. (a) Low-pass prototype. (b) Conversion of series inductors to shunt capacitors. (c) Low-pass to bandpass transformation. (d) TLs approximations of parallel resonators and $J$ inverters.

impedance at the input and output ports of the filter are specified during the filter design. The low-pass-to-bandpass transformation of Fig. 7(b) then leads directly to Fig. 7(c), consisting of the $N$ shunt $L-C$ resonators with $N-1$ series $J$ inverters [36]. Finally, the TL elements are applied to realize the parallel resonator and $J$ inverters, forming a TL BPF [35]. Notably, $Z_{c i}, Z_{L i}, F_{c i}$, and $F_{L i}$, shown in Fig. 7(d), are the same as those defined in Fig. 5(b).

Following the above-mentioned procedure, Fig. 8 presents a practical example of a third-order BPF with detailed design parameters. The circuit is also simulated using the ideal TL model, neglecting junction effects. Fig. 9 plots the corresponding frequency responses based on $50-\Omega$ reference impedance. Four transmission zeros are set at 1.78, 1.88, 3.19, and $3.95 \mathrm{GHz}$, forming a passband with a center frequency $\left(f_{0}\right)$ at $2.5 \mathrm{GHz}$. 


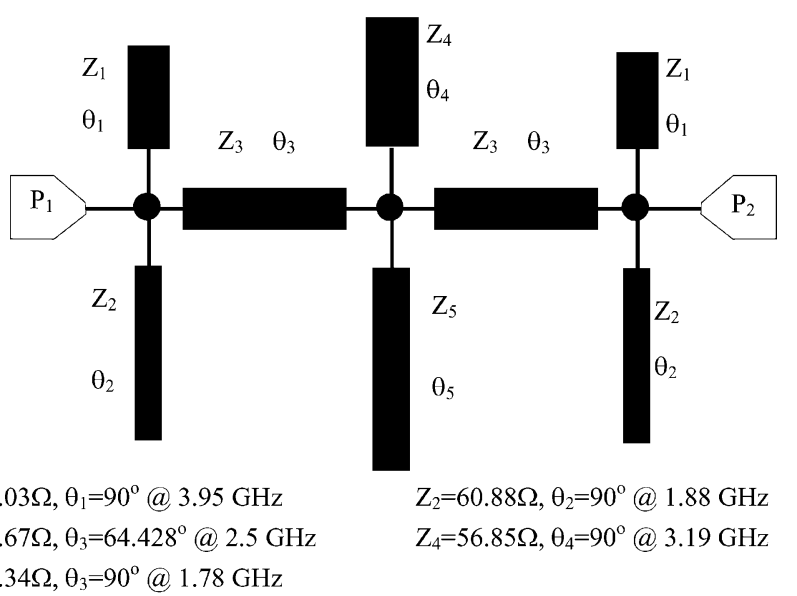

Fig. 8. Equivalent circuit of a $2.5-\mathrm{GHz}$ idealized TL BPF.

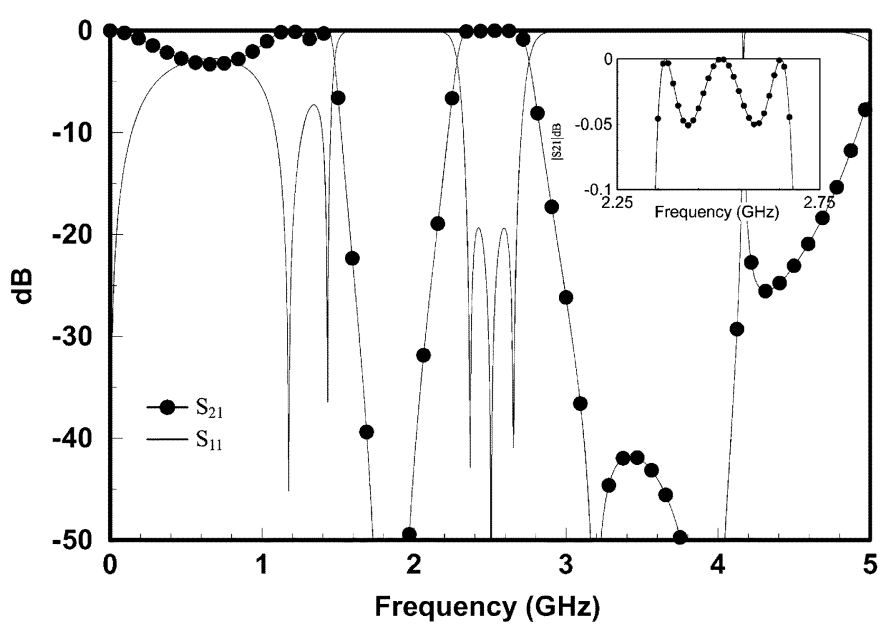

Fig. 9. Frequency responses of idealized TL BPF neglecting junction effects.

Therefore, the out-band rejection can be below $35 \mathrm{~dB}$ from 3.1 to $4.15 \mathrm{GHz}$. The return loss is below $-19.429 \mathrm{~dB}$ from 2.329 to $2.671 \mathrm{GHz}$, achieving a passband with an equal ripple of $0.05 \mathrm{~dB}$. Notably, the BPF shown in Fig. 8 is symmetrical and directly matches the $50-\Omega$ system without further impedance transformation.

\section{Miniaturized TL BPF: Layout and Measurements}

The practical implementation of a miniaturized BPF by mapping the idealized BPF shown in Fig. 8 to the four-layer stacked CCS TL filter configuration is presented here. Fig. 10 shows the three-dimensional view of the miniaturized TL BPF, incorporating a multilayer CCS TL. The CCS TL is realized by a unit cell with a period of $0.35 \mathrm{~mm}(P=0.35 \mathrm{~mm})$ and is realized in a four-layer print circuit board (PCB). The permittivity and thickness of each substrate are 4.7 and $0.06 \mathrm{~mm}$ with a loss tangent of 0.013. All metal layers are copper with a thickness of $0.0175 \mathrm{~mm}$. The guiding characteristics of CCS TLs, including the propagation constants and characteristic impedances, are extracted from the theoretical $S$-parameters, which are calculated by the full-wave EM simulator [31]. The extracted data are applied to define the width and meandered shapes of the CCS TLs in different layers. As shown in Fig. 10, five TLs, including two series TLs with an electrical length of $64.428^{\circ}$ at $2.5 \mathrm{GHz}$, two

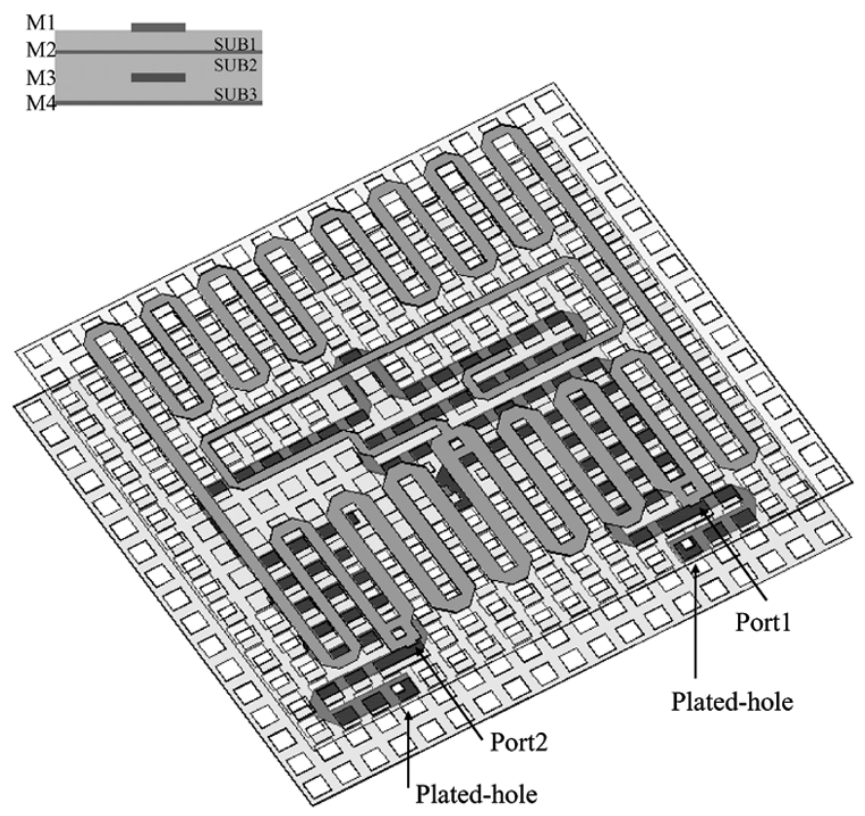

Fig. 10. Three-dimensional view of a 2.5-GHz TL BPF.

$90^{\circ}$ shunt stubs at $1.88 \mathrm{GHz}$, and one $90^{\circ}$ shunt stub at $3.19 \mathrm{GHz}$ are in the $\mathrm{M} 1$ and $\mathrm{M} 2$ metal layers. Additionally, two $90^{\circ} \mathrm{TLs}$ at $3.95 \mathrm{GHz}$ and one $90^{\circ} \mathrm{TL}$ at $1.78 \mathrm{GHz}$ are realized using sandwiched CCS TLs in M2-M4 metal layers. The minimum and maximum linewidths are 0.11 and $0.18 \mathrm{~mm}$, respectively. The reference ground planes (M2 and M4) of the four-layer configuration are connected by plated holes filled with copper for proper grounding. Two external terminals of the BPF are located on the M1 layer, facilitating the interface to the probe tips.

The device-under-test (DUT) is very thin and small so measurements cannot be easily made using coaxial connectors or cables. Therefore, two 50- $\Omega$ ground-signal-ground (G-S-G) coplanar-waveguide (CPW)-based microwave probes from Picoprobe, Naples, FL, are applied to make the measurements. The chuck, which is a metal plate for supporting the DUT, is grounded to the instruments. Therefore, a piece of paper with a thickness of $0.05 \mathrm{~mm}$ is inserted between the DUT and the chuck for proper isolation.

Before the measurements are made, the whole system, including an Agilent 8510C VNA, cables, and probes is calibrated by performing two-port short-open-load-through (SOLT) procedure with CS-11 standard substrates from Picoprobe. Fig. 11 compares the measured and theoretical results. The theoretical data include the effects of the junctions, grounding vias, plated through-holes, finite conductivity, and dielectric losses.

The measured data shows four transmission zeros at 1.85, 1.98, 3.19, and $3.95 \mathrm{GHz}$. Notably, the low-side transmission zeros are shifted by approximately $5 \%(70 \mathrm{MHz})$ from the frequency responses shown in Fig. 9. The center frequency of the BPF is slightly shifted from 2.5 to $2.55 \mathrm{GHz}$ by approximately $2 \%$. However, Fig. 11 shows that the out-band rejection is highly consistent with the theoretical values predicted by simulation, remaining below $35 \mathrm{~dB}$ from 3.1 to $4.15 \mathrm{GHz}$. On the other hand, the measured return loss is below $-16.8 \mathrm{~dB}$ from 2.38 to $2.78 \mathrm{GHz}$ in the passband, and exceeds the simulated value by $3.05 \mathrm{~dB}$. Notably, the three reflection zeros are presented 


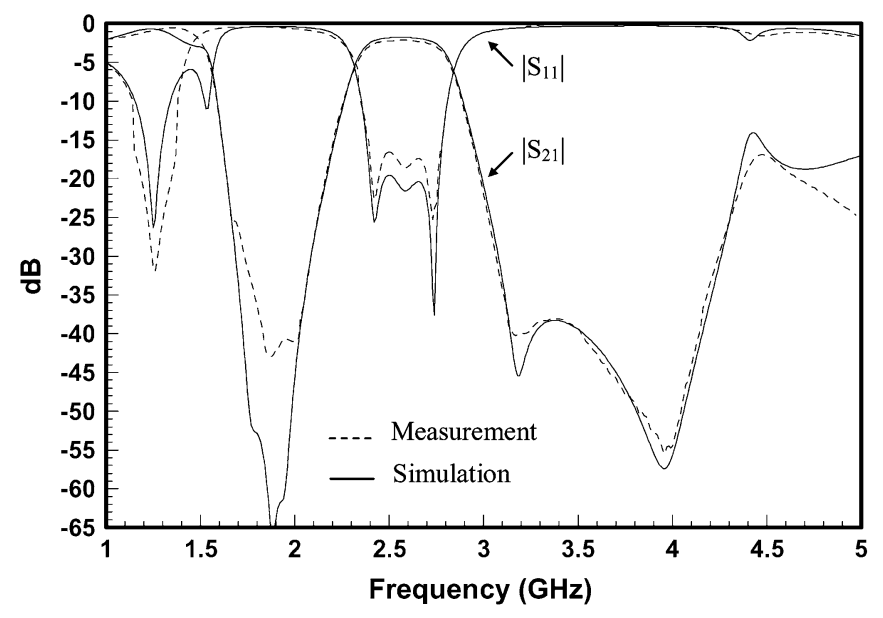

Fig. 11. Measured results of miniaturized TL BPF.

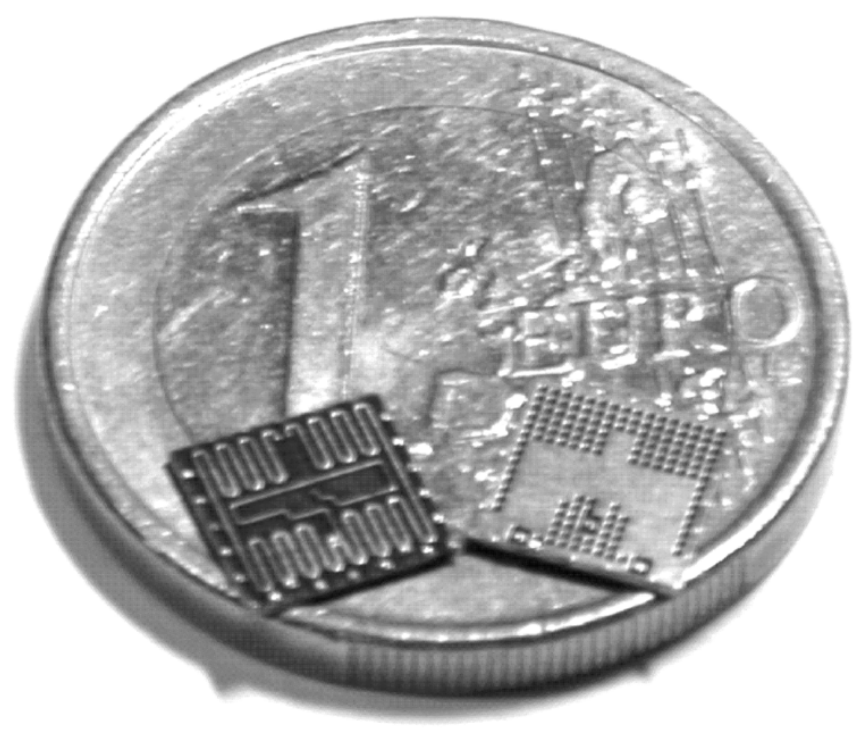

Fig. 12. 2.5-GHz miniaturized BPF on one Euro $(\epsilon 1)$.

at approximately $2.42,2.58$, and $2.74 \mathrm{GHz}$, offset by only $1 \%$ against the idealized frequency responses shown in Fig. 9. The measured insertion loss is approximately $2.46 \mathrm{~dB}$ from 2.38 to $2.78 \mathrm{GHz}-0.4 \mathrm{~dB}$ higher than the simulated value. Fig. 12 shows the photograph of the prototype, whose dimensions are $5.0 \times 5.0 \times 0.18 \mathrm{~mm}$.

\section{DISCUSSION}

The miniaturized filter is implemented solely using the stacked CCS TLs so the total volume of the filter can be expressed by the following equation:

$$
V_{\text {total }}=L_{t} \cdot P \cdot\left(2-\frac{1}{N}\right) \cdot h .
$$

$L_{t}$ is the total length of the all of the TLs in the filter design. $P$ is the period of the unit cell of the CCS TL. $N$ is the number of signal layers in the multilayer system configuration and $h$ is the thickness of single-layer substrate. Therefore, the number of substrates is $2 N-1$.
TABLE I

VARIABLES FOR VOLUME ESTIMATION OF PROPOSED BPF

\begin{tabular}{l|l}
\hline Variable & \multicolumn{1}{c}{ Value } \\
\hline$f_{0}$ & $2.5 \mathrm{GHz}$ \\
\hline$N_{z}$ & $4(1.78 \mathrm{GHz}, 1.88 \mathrm{GHz}, 3.19 \mathrm{GHz}, 3.95 \mathrm{GHz})$ \\
\hline$N$ & 2 \\
\hline$h$ & $0.06 \mathrm{~mm}$ \\
\hline$\varepsilon_{r}$ & 4.7 \\
\hline$P$ & $0.35 \mathrm{~mm}$ \\
\hline$L_{t}$ & $115.1 \mathrm{~mm}^{3}$ \\
\hline $\mathrm{V}_{\text {estimate }}$ & $3.63 \mathrm{~mm}^{3}$ \\
\hline $\mathrm{V}_{\text {prototype }}$ & $4.5 \mathrm{~mm}^{3}$ \\
\hline
\end{tabular}

$N_{z}$ is the number of transmission zeros with a minimum value of two. In the first-order approximation, $L_{t}$ is inversely proportional to the square root of the relative dielectric constant $\left(\sqrt{\varepsilon_{r}}\right)$. With reference to Fig. 8, the total volume of the three-order 2.5-GHz BPF (with four transmission zeros) can be estimated using (2). Table I lists the relevant design parameters, showing good agreement between hand calculations and prototype dimensions. Notably, the parameters including $P, h, \varepsilon_{r}$, and $N$ are process related and all in typical values for the current PCB technology. On the other hand, $L_{t}$ and $N_{z}$ are related to electric specifications of the proposed miniaturization that incorporates the meandered CCS TL to systematically reduce the volume of BPF, which approaches the limits of state-of-art technology. The estimated $V_{\text {total }}$ is $19 \%$ less than that of the prototype so the approach based on the proposed stacked meandered CCS TL can effectively miniaturize microwave passive circuits such as the BPF presented here. With reference to Fig. 1, the size of the proposed prototype also approaches that of state-of-art technology-approximately $4.5 \mathrm{~mm}^{3}$. In the circumstance, when a designer requires that the area is smaller than that achieved using the presented four-layer prototype, $N$ may be increased from two to four. By doing so, the area will be changed from $5.0 \mathrm{~mm} \times 5.0 \mathrm{~mm}$ to $1.83 \mathrm{~mm} \times 1.83 \mathrm{~mm}$. The thickness will be increased from 0.18 to $1.26 \mathrm{~mm}$, and the volume will be changed from 4.5 to $4.23 \mathrm{~mm}^{3}$. Equation (2) also clearly shows that when $P$ and $h$ are reduced, the total volume is scaled down to an extent proportional to the product of $P$ and $h$. Also, $P$ stands for the periodicity of the unit cell and is the limit on the line pitch, which is the center-to-center distance between the parallel lines associated with particular processes.

\section{CONCLUSION}

This paper has proposed a novel approach for designing a synthetic quasi-TEM TL stacked in a multilayer substrate configuration. The synthetic TLs are formed by alternating the so-called CCS quasi-TEM lines in different signal layers separated by the meshed ground planes, whose isolation property has been experimentally verified to a satisfactory level of negligible effects on desired circuit performances. The multilayer synthetic TL has been successfully applied to the design of a 2.4-GHz ISM-band BPF prototype of a size approaching that of the state-of-the-art device. The proposed guiding structures can be extended to the design of a SIP and RF SOC, when miniaturization becomes critical. 


\section{REFERENCES}

[1] T. Tsujiguchi, H. Matsumoto, and T. Nishikawa, "A miniaturized double-surface CPW bandpass filter improved spurious responses," IEEE Trans. Microw. Theory Tech., vol. 49, no. 5, pp. 879-885, May 2001

[2] L. K. Yeung and K.-L. Wu, "A compact second-order LTCC bandpass filter with two finite transmission zeros," IEEE Trans. Microw. Theory Tech., vol. 51, no. 2, pp. 337-341, Feb. 2003.

[3] C.-W. Tang, Y.-C. Lin, and C.-Y. Chang, "Realization of transmission zeros in combline filters using an auxiliary inductively coupled ground plane," IEEE Trans. Microw. Theory Tech., vol. 51, no. 10, pp. 2112-2118, Oct. 2003.

[4] C.-W. Tang, "Harmonic-suppression LTCC filter with the stepimpedance quarter-wavelength open stub," IEEE Trans. Microw. Theory Tech., vol. 52, no. 2, pp. 617-624, Feb. 2004.

[5] Y. W. Kong and S. T. Chew, "EBG-based dual mode resonator filter," IEEE Microw. Wireless Compon. Lett., vol. 14, no. 3, pp. 124-126, Mar. 2004

[6] Y.-K. Kuo, C.-H. Wang, and C. H. Chen, "Novel reduced-size coplanarwaveguide bandpass filters," IEEE Microw. Wireless Compon. Lett., vol. 14 , no. 3, pp. 65-67, Feb. 2001.

[7] J.-T. Kuo, M.-J. Maa, and P.-H. Lu, "A microstrip elliptic function filter with compact miniaturized hairpin resonators," IEEE Microw. Wireless Compon. Lett., vol. 10, no. 3, pp. 94-95, Mar. 2000.

[8] K.-C. Huang, D. Hyland, A. Jenkins, D. Edwards, and D. Dew-Hughes, "A miniaturized interdigital microstrip bandpass filter," IEEE Trans. Appl. Supercond., vol. 9, no. 2, pp. 3889-3892, Jun. 1999.

[9] G. S. Ching, "Design and practical implementation of a $2.4 \mathrm{GHz}$ lumped element elliptic bandpass filter," in Proc. Asia-Pacific Microwave Conf., Taipei, Taiwan, R.O.C., 2001, pp. 523-526.

[10] A. Görür, "Description of coupling between degenerate modes of a dualmode microstrip loop resonator using a novel perturbation arrangement and its dual-mode bandpass filter applications," IEEE Trans. Microw. Theory Tech., vol. 52, no. 2, pp. 671-677, Feb. 2004

[11] L. Zhu and B. C. Tan, "Miniaturized dual-mode bandpass filter using inductively loaded cross-slotted patch resonator," IEEE Microw. Wireless Compon. Lett., vol. 15, no. 1, pp. 22-24, Jan. 2005.

[12] M. G. Banciu, R. Ramer, and A. Ioachim, "Compact microstrip resonators for $900 \mathrm{MHz}$ frequency band," IEEE Microw. Wireless Compon. Lett., vol. 13, no. 5, pp. 175-177, May 2003.

[13] J. Zhou, M. J. Lancaster, and F. Huang, "HTS coplanar meander-line resonator filters with a suppressed slot-line mode," IEEE Trans. Appl. Supercond., vol. 14, no. 1, pp. 28-32, Mar. 2004.

[14] K. Entesari, T. Vaha-Heikkila, and G. M. Rebeiz, "Miniaturized differential filters for $C$ - and $K u$-band applications," in Proc. Eur. Microwave Conf., Munich, Germany, 2003, pp. 227-230.

[15] E. Goron, J. P. Coupez, C. Person, Y. Toutain, H. Lattard, and F. Perrot, "Accessing to UMTS filtering specifications using new microstrip miniaturized loop-filters," in IEEE MTT-S Int. Microwave Symp. Dig., 2003 , pp. 1599-1602.

[16] N. Ishitobi, E. Ajioka, T. Abe, and H. Ninomiya, "The improvement of miniaturized multilayer ceramic bandpass filter," in IEEE MTT-S Int. Microwave Symp. Dig., 1999, pp. 1343-1346.

[17] J. S. Hong and M. J. Lancaster, "Recent advances in microstrip filters for communications and other applications," in IEE Advances Passive Microwave Components Dig., 1997, pp. 2/1-2/6.

[18] R. S. Kwok, S. J. Fiedziuszko, F. A. Miranda, G. V. Leon, M. S. Demo, and D. Y. Bohman, "Miniaturized HTS/dielectric multilayer filters for satellite communications," IEEE Trans. Appl. Supercond., vol. 7, no. 2, pp. 3706-3709, Jun. 1997.

[19] H.-K. Zeng, A. Hsiao, W.-H. Hsu, S.-W. Wu, J.-Y. Lin, K.-H. Wu, J.-Y. Juang, T.-M. Uen, Y.-S. Gou, and J.-T. Kuo, "Miniaturized $3 \mathrm{GHz}$ crosscoupled planar microwave filters," IEEE Trans. Appl. Supercond., vol. 14, no. 1, pp. 107-111, Mar. 2004.

[20] C.-S. Kim, J.-S. Lim, J.-H. Kim, and D. Ahn, "A design of a miniaturized 2-pole bandpass filter by using a slot and a hair-pin line," in IEEE MTT-S Int. Microwave Symp. Dig., 2004, pp. 1983-1986.

[21] Y. Toutain, J.-P. Coupez, and C. Person, "Microstrip miniaturized loopfilters with high out-of-band rejection for future 3G mobile terminals," in IEEE MTT-S Int. Microwave Symp. Dig., 2001, pp. 1589-1592.

[22] S.-Y. Lee and C.-M. Tsai, "New cross-coupled filter design using improved hairpin resonators," IEEE Trans. Microw. Theory Tech., vol. 48, no. 12, pp. 2482-2490, Dec. 2000.

[23] T. Tsujiguchi, H. Matsumoto, and T. Nishikawa, "A miniaturized endcoupled bandpass filter using $\lambda / 4$ hair-pin coplanar resonators," in IEEE MTT-S Int. Microwave Symp. Dig., 1998, pp. 829-832.
[24] W. Diels, K. Vaesen, P. Wambacq, S. Donnay, W. De Raedt, M. Engels, and I. Bolsens, "Single-package integration of RF blocks for a 5 GHz WLAN application," IEEE Trans. Adv. Packag., vol. 24, no. 3, pp. 384-391, Aug. 2001.

[25] C.-H. Lee, A. Sutono, S. Han, K. Lim, S. Pinel, E. M. Tentzeris, and J Laskar, "A compact LTCC-based $K u$-band transmitter module," IEEE Trans. Adv. Packag., vol. 25, no. 3, pp. 374-384, Aug. 2002.

[26] J. Ryckaert, S. Brebels, B. Come, W. Diels, D. Hauspie, S. Stoukatch, K. Vaesen, W. De Raedt, and S. Donnay, "Single-package 5 GHz WLAN RF module with embedded patch antenna and $20 \mathrm{dBm}$ power amplifier," IEEE MTT-S Int. Microwave Symp. Dig., pp. 1037-1040, 2003.

[27] Y.-S. Lin, C.-C. Liu, K.-M. Li, and C. H. Chen, "Design of an LTCC tri-band transceiver module for GPRS mobile applications," IEEE Trans. Microw. Theory Tech., vol. 52, no. 12, pp. 2718-2724, Dec. 2004.

[28] M. M. Tentzeris, J. Laskar, J. Papapolymerou, S. Pinel, V. Palazzari, R. Li, G. Dejean, N. Papageorgiou, D. Thompson, R. Bairavasubramanian, S. Sarkar, and J.-H. Lee, "3-D-integrated RF and millimeter-wave functions and modules using liquid crystal polymer (LCP) system-on-package technology," IEEE Trans. Adv. Packag., vol. 27, no. 2, pp. 332-340, May 2004.

[29] A. Chernyakov, K. Markov, D. Orlenko, P. Heide, and C. Ruppel, "Miniature fully-integrated WLAN frontend-modules based on LTCC technology," in IEEE MTT-S Int. Microwave Symp. Dig., 2004, pp. 139-142.

[30] A. C. W. Lu, K. M. Chua, L. L. Wai, S. C. K. Wong, J. J. Wang, and Y. P. Zhang, "Integrated antenna module for broad-band wireless applications," in Electronics Packaging Tech. Conf., 2004, pp. 240-243.

[31] C.-C. Chen and C.-K. C. Tzuang, "Synthetic quasi-TEM meandered transmission lines for compacted microwave integrated circuits," IEEE Trans. Microw. Theory Tech., vol. 52, no. 6, pp. 1637-1647, Jun. 2004.

[32] C. Quendo, E. Rius, and C. Person, "Narrow bandpass filters using dualbehavior resonators," IEEE Trans. Microw. Theory Tech., vol. 51, no. 3, pp. 734-743, Mar. 2003

[33] — "Narrow bandpass filters using dual-behavior resonators based on stepped-impedance stubs and different-length stubs," IEEE Trans. Microw. Theory Tech., vol. 52, no. 3, pp. 1034-1044, Mar. 2004.

[34] G. Prigent, E. Rius, F. L. Pennec, S. L. Maguer, C. Quendo, G. Six, and H. Happy, "Design of narrow-band DBR planar filters in Si-BCB technology for millimeter-wave applications," IEEE Trans. Microw. Theory Tech., vol. 52, no. 3, pp. 1045-1051, Mar. 2004.

[35] H.-S. Wu, R. H. Yang, and C.-K. C. Tzuang, "Synthesis of quasi-elliptical fishbone bandpass filter," in Proc. Asia-Pacific Microwave Conf., New Delhi, India, 2004, Post Section 2.

[36] G. L. Matthaei, L. Young, and E. M. T. Jones, Microwave Filters, Impedance-Matching Networks, and Coupling Structures. Dedham, MA: Artech House, 1980.

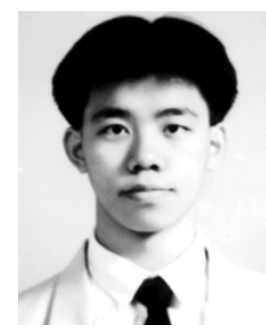

Hsien-Shun Wu (S'97-M'05) received the B.S. degree in electronic engineering from National Taipei University of Technology, Taipei, Taiwan, R.O.C., in 1999, the M.S. degree in communication engineering from National Chiao Tung University, Hsinchu, Taiwan, R.O.C., in 2001, and is currently working toward the Ph.D. degree in communication engineering at National Chiao Tung University.

His research interests include design of wireless system modules and design and development of photonic-bandgap (PBG) structures for RF circuits.

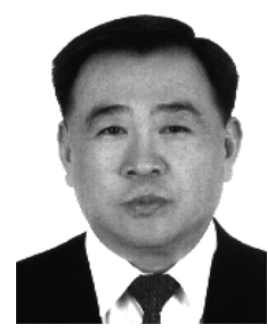

Houng-Jay Yang received the M.S. degree in physics from Southern Methodist University, Dallas, TX, in 1975, and the Engineer degree in electrical engineering from the University of California at Los Angeles, in 1979

Upon graduation, he joined TRW, Redondo Beach, CA, where he developed microwave circuits. In 1986, he joined Hughes Aircraft Satellite System Division, El Segundo, CA, and later became a Scientist involved in the contribution of the development of passive modules in multiple geostationary satellite payloads. From 2002 to 2003, he was an Invited Professor with National Chiao Tung University, Hsinchu, Taiwan, R.O.C. He is currently a consultant in the U.S. 


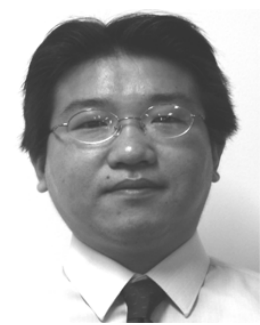

Ching-Juang Peng was born in Hsinchu, Taiwan, R.O.C., 1974. He received the B.S. degree in mechanical manufacturing engineering from the National Yunlin Industrial Junior College, Yunlin, Taiwan, R.O.C., in 1996.

From 1998 to 2000, he was with the TriCOME Microwave Electronic Corporation, Hsinchu, Taiwan, R.O.C., where he was involved with monolithic microwave integrated circuit (MMIC) packages and surface-mounted antennas. From 2000 to 2003, he was with National Chiao Tung University, where he was in charged of the assembling and manufacturing of millimeter-wave wireless transceiver modules. Since 2003, he has been with Epic Communication Inc., Hsinchu, Taiwan, R.O.C. His interests include the design of system-in-packages (SIP) and compact antennas for mobile communications.

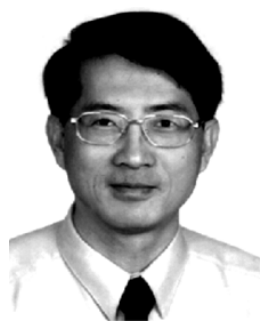

Ching-Kuang C. Tzuang (S'80-M'80-SM'92F'99) received the B.S. degree in electronic engineering from National Chiao Tung University, Hsinchu, Taiwan, R.O.C., in 1977, the M.S. degree from the University of California at Los Angeles, in 1980, and the Ph.D. degree in electrical engineering from the University of Texas at Austin, in 1986.

From 1981 to 1984, he was with TRW, Redondo Beach, CA, where he was involved with analog and digital MMICs. Since 1986, he has been with the Institute of Communication Engineering, National Chiao Tung University. In February 2004, he joined the Graduate Institute of Communication Engineering, Department of Electrical Engineering, National Taiwan University, Taipei, Taiwan, R.O.C., where he continues his research on advanced guiding structures for research and development of RF system-on-a-chip, integrating active and passive microwave/millimeter-wave RF signal-processing components into a single chip. His research activities also involve the design and development of millimeter-wave and microwave active and passive circuits and the field theory analysis and design of various complex-waves guiding structures and large-array antennas. He has supervised 61 M.S. students and $21 \mathrm{Ph} . \mathrm{D}$. students.

Dr. Tzuang helped in the formation of the IEEE Microwave Theory and Techniques Society (IEEE MTT-S) Taipei Chapter, and served as secretary, vice chairman, and chairman in 1988, 1989, and 1990, respectively. He has been on the Asia-Pacific Microwave Conference International Steering Committee, where, since 1994, he has represented the Taipei Chapter as the international liaison officer. 